\title{
PA-009 EFFICACY AND TOLERABILITY OF REPEATED ADMINISTRATION OF ACTS OVER A PERIOD OF TWO YEARS IN CHILDREN AND ADULT PATIENTS WITH ACUTE UNCOMPLICATED MALARIA IN BURKINA FASO
}

Issiaka Soulama, Moïse Kaboré, Aboubacar Coulibaly, Maurice Ouattara, Souleymane Sanon, Amidou Diarra, Edith Bougouma, Alphonse Ouedraogo, Benjamin Sombie, Amidou Ouedraogo, Désiré Kargougou, Daouda Ouattara, Nebie Issa, Alfred Tiono, Sodiomon Sirima. CNRFP, Burkina Faso

\subsection{6/bmjgh-2016-000260.48}

Background According to the guidelines of the Burkina Faso National Malaria Control Programme, artesunate-amodiaquine (ASAQ) and artemether-lumefantrine (AL) are the first-line drugs for uncomplicated malaria treatments. However, in some contexts where individuals will experience more than 1 episode of clinical malaria per year, it is unknown to what extent giving any of these ACTs repeatedly is safe. In the framework of the activities of the West African Network for Antimalarial Drugs (WANECAM) network, we have compared the efficacy and tolerability of repeated use of dihydroartemisinin-piperaquine (DHA-PQ) or artesunatepyronaridine (PYR) with artesunate-amodiaquine (ASAQ).

Methods A randomised open-label parallel 3 arms trial was conducted to compare the efficacy of a three-day regimen of DHA-PQ and PYR with ASAQ. The trial involved children and adults with uncomplicated falciparum malaria. Participants were randomly assigned to one of the three treatment arms at the first clinical episode. During the subsequent clinical episodes, the same drug was administered. Follow-up duration was 42 days for each episode. Study duration was two years for each participant. Primary endpoints were the incidence rate of uncomplicated malaria over a period of 2 years and PCR corrected/uncorrected ACPR at day 28 and day 42. Safety parameters were also assessed. 
Results Of the 763 patients enrolled, the incidence rate of clinical malaria was $1.4,1.2$, and 1.5 episodes / person-year at risk in the ASAQ, DHA-PQ and PYRAMAX arms, respectively. The PCR-uncorrected efficacy at day 28 versus day 42 was: ASAQ 93.4\% vs 79.5\%; PYR 98.1\% vs 74.8\%; and DHA-PQ 99.5\% vs 95.2\%. Bronchitis, rhinitis, abdominal pain, cough, QTc prolongation, headache, and vomiting were registered as the main adverse events in each of the three groups.

Conclusions The findings from our study support the current recommendations for using artemisinin-based combinations in the treatment of uncomplicated malaria in areas of high malaria transmission such as Burkina Faso. 